\title{
Synthesis of (pyrimidin-4-yloxy)- and (pyrimidin-3-yl)acetyl azides and their rearrangement to carbamates and ureas
}

\author{
Virginija Jakubkiene,* Zana Kacnova, Milda M. Burbuliene, and Povilas Vainilavicius \\ Department of Organic Chemistry, Faculty of Chemistry, Vilnius University \\ Naugarduko 24, LT-03225, Vilnius, Lithuania \\ E-mail: virginija.jakubkiene@chf.vu.lt
}

\begin{abstract}
On nitrosation of (pyrimidin-4-yloxy)- and (pyrimidin-3-yl)acetohydrazides 2, 3 with sodium nitrite in diluted hydrochloric acid at $0-5{ }^{\circ} \mathrm{C}$ the corresponding (pyrimidinyl)acetyl azides 5, 6 were prepared. Azides 5, 6 undergo Curtius rearrangement in the reactions with alcohols, phenols, arylamines or water to give carbamates 7-9, 11-14 or ureas 15-21.
\end{abstract}

Keywords: (Pyrimidinyl)acetyl azide, (pyrimidinyl)methylcarbamate, (pyrimidinyl)methylurea, nitrosation, Curtius rearrangement

\section{Introduction}

In recent years naturally occurring and synthetic heterocyclic compounds are of interest in pharmaceutical and pesticide research. ${ }^{1}$ Pyrimidines, in particular, cover the considerable position among compounds exhibiting diverse biological activities. ${ }^{2-6}$ On the other hand, compounds with the linkage of carbamate or urea are citied to show cytotoxic, ${ }^{7-12}$ antimicrobial and insecticidal ${ }^{13}$ or fatty acid amide hydrolase inhibitory ${ }^{14}$ activity. There are some recent examples of biologically active pyrimidine-based carbamates and ureas. ${ }^{15-19}$

Carbamates and ureas are frequently synthesized from acyl azides via Curtius rearrangement. ${ }^{13,20}$ A number of methods for the synthesis of acyl azides have been reported. Acyl azides are commonly prepared from carboxylic acid and ethyl chloroformate via mixed anhydrides and sodium azide or by the reaction of acyl chlorides with trimethylsilyl azide. ${ }^{21} \mathrm{~A}$. R. Katritzsky et al. recently reported a simple and safe synthesis of acyl azides from the corresponding $\mathrm{N}$-acyl benzotriazoles and sodium azide. ${ }^{22}$ Acyl azides can also be prepared from hydrazides by nitrozation with sodium nitrite in acidic media. ${ }^{13,20,23}$ This approach is a convenient one, especially for readily accessible hydrazides. Hydrazide nitrosation to give acyl azides is applied for a large-scale synthesis. ${ }^{24}$ 
Continuing our interest in the synthesis of compounds with potential biological activity, ${ }^{6,25}$ herein we report the synthesis of new pyrimidine-based acetyl azides and their rearrangement reactions to form carbamates and ureas.

\section{Results and Discussion}

Previously we reported the synthesis of acetyl azide $\mathbf{4}$ by nitrosation of hydrazide 1 in a $50 \%$ acetic acid or diluted hydrochloric acid. ${ }^{26}$ Performing this reaction in acetic acid the yield of azide $\mathbf{4}$ was higher, when in a diluted hydrochloric acid together with the azide $\mathbf{4}$ formation some azide 6 was obtained. In this work azides 5, 6 were successfully prepared under treatment of acetohydrazides 2, 3 with sodium nitrite at $0-5{ }^{\circ} \mathrm{C}$ in a diluted hydrochloric acid solution (Scheme 1).<smiles>NNC(=O)CBr</smiles>

$1-3$<smiles>CSc1nc(C)cc(=O)n1C</smiles>

1,4
$\mathrm{HNO}_{2}$

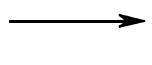<smiles>[10B]CC([NH])=O</smiles>

4-6<smiles>COc1cc(C)nc(SC)n1</smiles>

2,5<smiles>Cc1cc(=O)n(C)c(=O)[nH]1</smiles>

3,6

\section{Scheme 1}

Compounds 5, 6 were sufficiently pure to be used for further rearrangement reactions. The IR spectra of acyl azides 5, 6 display strong absorption bands characteristic for azide stretching vibrations at 2158 and $2150 \mathrm{~cm}^{-1}$, respectively.

Acyl azides undergo thermal rearrangement in an inert solvents to give isocyanates (Curtius rearrangement), which on reaction with alcohols or amines lead to the formation of carbamates

and ureas. $^{21}$ Azide 5 being heated at reflux in benzene with phenols gave carbamates 7-9 (Scheme 2). 


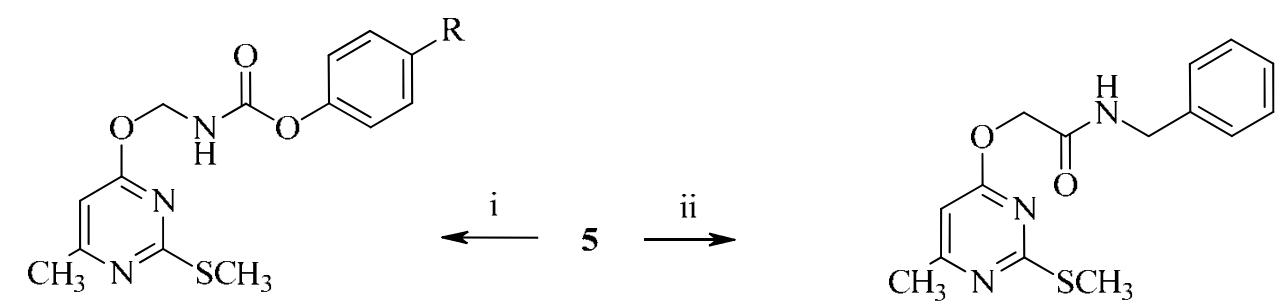

7-9

$\mathrm{R}=\mathrm{H}(\mathbf{7}), \mathrm{Cl}(\mathbf{8}), \mathrm{F}(\mathbf{9})$.

i: 4-R-C $\mathrm{C}_{6} \mathrm{H}_{4}-\mathrm{OH}$, dry $\mathrm{C}_{6} \mathrm{H}_{6} ;$ ii: $\mathrm{C}_{6} \mathrm{H}_{5} \mathrm{CH}_{2} \mathrm{NH}_{2}$, dry $\mathrm{C}_{6} \mathrm{H}_{6}$.

\section{Scheme 2}

Analytically pure carbamates 7-9 after recrystallization from 2-propanol were isolated in 43$59 \%$ yields. The reaction of azide 5 and benzylamine to form the corresponding urea was unsuccessful, amide $\mathbf{1 0}$ was isolated instead. Heating of azide 5 at reflux in benzene for $4 \mathrm{~h}$ to rearrange to isocyanate, then addition of benzylamine and further heating for $1 \mathrm{~h}$ gave a complex mixture of unidentified products. By adding azide 5 and benzylamine at one go and immediate heating in benzene for $1 \mathrm{~h}$ resulted in amide $\mathbf{1 0}$ formation in $67 \%$ yield. The ${ }^{1} \mathrm{H}$ NMR spectra of carbamates 7-9 show characteristic dublets at 5.50 and $5.51 \mathrm{ppm}$ for $\mathrm{OCH}_{2}$ group protons and triplets for $\mathrm{NH}$ protons (broad singlet for comp. 8) in the region of 6.53-6.56 ppm. In the ${ }^{1} \mathrm{H}$ NMR of amide 10 singlet at $4.95 \mathrm{ppm}$ for $\mathrm{OCH}_{2}$ group protons, dublet at $4.56 \mathrm{ppm}$ for $\mathrm{CH}_{2}$ benzyl protons and broad singlet at $6.60 \mathrm{ppm}$ due to $\mathrm{NH}$ proton were observed.

In contrast to azide 5, reactions of azide $\mathbf{6}$ with nucleophiles proceeded smoothly and yields of products were higher (Scheme 3). 
<smiles>COC(=O)NCn1c(=O)cc(C)[nH]c1=O</smiles>

11

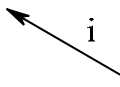

iii

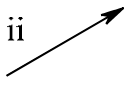

6

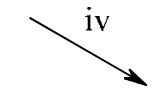<smiles>[R]c1ccc(OC(=O)NCn2c(=O)cc(C)[nH]c2=O)cc1</smiles>

12-14<smiles>[R]c1ccc(NC(=O)NCn2c(=O)cc(C)[nH]c2=O)c([R])c1[R]</smiles>

15-20<smiles>Cc1cc(=O)n(CNC(=O)NCn2c(=O)cc(C)[nH]c2=O)c(=O)[nH]1</smiles>

21

$\mathrm{R}=\mathrm{H}$ (12), Cl (13), F (14); $\mathrm{R}^{\mathrm{l}}=\mathrm{R}^{2}=\mathrm{R}^{3}=\mathrm{H}(\mathbf{1 5}) ; \mathrm{R}^{\mathrm{l}}=\mathrm{CH}_{3}, \mathrm{R}^{2}=\mathrm{R}^{3}=\mathrm{H}(\mathbf{1 6}) ; \mathrm{R}^{\mathrm{l}}=\mathrm{R}^{2}=\mathrm{H}, \mathrm{R}^{3}=\mathrm{OCH}_{3}$ (17);

$\mathrm{R}^{\mathrm{l}}=\mathrm{R}^{2}=\mathrm{H}, \mathrm{R}^{3}=\mathrm{F}(\mathbf{1 8}) ; \mathrm{R}^{\mathrm{l}}=\mathrm{R}^{3}=\mathrm{Cl}, \mathrm{R}^{2}=\mathrm{H}(\mathbf{1 9}) ; \mathrm{R}^{\mathrm{l}}=\mathrm{R}^{3}=\mathrm{H}, \mathrm{R}^{2}=\mathrm{CF}_{3}(\mathbf{2 0})$.

i: abs. $\mathrm{CH}_{3} \mathrm{OH}$; ii: $\mathrm{R}-\mathrm{OH}$, dry dioxane; iii: $\mathrm{R}^{3}-\mathrm{NH}_{2}$, dry dioxane; iv: $\mathrm{H}_{2} \mathrm{O}$, dioxane.

\section{Scheme 3}

Thus, azide 6 under heating at reflux for $5 \mathrm{~h}$ in methanol gave carbamate 11 in $56 \%$ yield. For reactions with phenols dry solvents, benzene and dioxane, were used. In benzene azide $\mathbf{6}$ did not show any reaction with phenols, likely because of poor solubility. In dioxane on heating at reflux for $5 \mathrm{~h}$ azide 6 with phenols furnished carbamates 12-14 in 63-74\% yields. In the ${ }^{1} \mathrm{H}$ NMR spectra of 11-14 the characteristic doublets for $\mathrm{NCH}_{2}$ group protons were observed at 5.09 and $5.18 \mathrm{ppm}$ along with the broad singlets in the region of 7.49 and $8.17-8.25 \mathrm{ppm}$. due to $\mathrm{NH}$ protons.

Heating at reflux of acyl azide 6 and arylamines in dioxane for $1 \mathrm{~h}$ produced ureas 15-20 in $57-88 \%$ yields, while heating at reflux for $4 \mathrm{~h}$ of azide 6 in the presence of water gave disubstituted urea 21. In the ${ }^{1} \mathrm{H}$ NMR spectra of ureas 15-20 characteristic dublets for $\mathrm{NCH}_{2}$ group protons resonate at $5.22-5.25 \mathrm{ppm}$ and the nearby $\mathrm{NH}$ proton shifts appear as triplets in the region of 6.78-7.86 ppm (for comp. 15 and 16 these peaks overlap with signals of aromatic protons). Besides the well-distinctive sharp one-proton peaks due to the $\mathrm{NH}$ group next to aromatic ring are observed downfield at 7.94-9.13 ppm. The symmetrical urea 21 spectrum shows a dublet at $5.10 \mathrm{ppm}$ for $\mathrm{NCH}_{2}$ group protons and neighboring $\mathrm{NH}$ proton as a triplet at $6.96 \mathrm{ppm}$ indicating magnetic equivalency of protons in both halves of the molecule.

The newly synthesized compounds were tested for antiproliferative activity in vitro. Compounds 7-21 were inactive. 
In conclusion, nitrosation of (pyrimidinyl)acetohydrazides is convenient and simple method for the synthesis of (pyrimidin-4-yloxy)- and (pyrimidin-3-yl)acetyl azides. Both of azides are suitable synthons for the new carbamate and urea synthesis.

\section{Experimental Section}

General. Melting points were determined in open capillaries and are uncorrected. NMR spectra were recorded on a Unity Varian INOVA spectrometer at $300 \mathrm{MHz}$ for ${ }^{1} \mathrm{H}$ and $75 \mathrm{MHz}$ for ${ }^{13} \mathrm{C}$. Chemical shifts $(\delta)$ are reported in ppm relative to TMS. The IR spectra were recorded on a Spectrum BX FT-IR (Perkin-Elmer, Sweden) as potassium bromide pellets. The reactions and purity of compounds was controlled by tlc on Silica gel $60 \mathrm{~F}_{254}$ plates (MERCK, Germany). Elemental analyses were performed at the Microanalytical Laboratory of the Department of Organic Chemistry of Vilnius University. All solvents were dried and distilled before use.

(6-Methyl-2-methylthiopyrimidin-4-yloxy)acetohydrazide 2 and (6-methyl-2,4-dioxo-1,2dihydropyrimidin-3(4H)-yl)acetohydrazide 3 were synthesized as reported in references ${ }^{27,28}$.

\section{General procedure for the synthesis of acetyl azides (5) and (6)}

To a cooled to $0-5{ }^{\circ} \mathrm{C}$ temperature suspension of hydrazide 2 or 3 (5 mmol), water $(15 \mathrm{~mL})$ and conc. hydrochloric acid $(4 \mathrm{~mL})$, a solution of sodium nitrite $(1.73 \mathrm{~g}, 25 \mathrm{mmol})$ in water $(10 \mathrm{~mL})$ was added dropwise under stirring. The reaction mixture was stirred at this temperature for $1 \mathrm{~h}$, the precipitate was collected by filtration (for compound 5 the reaction mixture was neutralized with sodium hydrogen carbonate before filtration), washed with cold water and dried over sodium sulphate in vacuum.

(6-Methyl-2-methylthiopyrimidin-4-yloxy)acetyl azide (3). Yield $0.88 \mathrm{~g}$ (74\%), mp 56-58 ${ }^{\circ} \mathrm{C}$; IR, v, $\mathrm{cm}^{-1}: 1706(\mathrm{C}=\mathrm{O}), 2158\left(\mathrm{~N}_{3}\right) ;{ }^{1} \mathrm{H} \mathrm{NMR}\left(\mathrm{CDCl}_{3}\right), \delta$, ppm: 2.42 (s, 3H, $\left.\mathrm{CH}_{3}\right), 2.51(\mathrm{~s}, 3 \mathrm{H}$, $\left.\mathrm{SCH}_{3}\right), 4.93\left(\mathrm{~s}, 2 \mathrm{H}, \mathrm{OCH}_{2}\right), 6.40(\mathrm{~s}, 1 \mathrm{H}, \mathrm{CH}) ;{ }^{13} \mathrm{C} \mathrm{NMR}\left(\mathrm{CDCl}_{3}\right), \delta, \mathrm{ppm}: 14.2,24.1,64.5$, 102.1, 168.0, 169.0, 171.6, 175.8. Anal. Calcd. for $\mathrm{C}_{8} \mathrm{H}_{9} \mathrm{~N}_{5} \mathrm{O}_{2} \mathrm{~S}: \mathrm{C}, 40.16 ; \mathrm{H}, 3.79 ; \mathrm{N}, 29.27$. Found: C, 40.52; H, 3.79; N, 29.43.

(6-Methyl-2,4-dioxo-1,2-dihydropyrimidin-3(4H)-yl)acetyl azide (6). Yield $0.74 \mathrm{~g}$ (71\%), mp 135-137 ${ }^{\circ} \mathrm{C}\left(\right.$ ref. $\left.^{26} \mathrm{mp} \mathrm{135-137}{ }^{\circ} \mathrm{C}\right)$; IR, v, $\mathrm{cm}^{-1}: 1634,1716,1741(\mathrm{C}=\mathrm{O}), 2150\left(\mathrm{~N}_{3}\right) ;{ }^{1} \mathrm{H} \mathrm{NMR}$ (DMSO-d $)_{6}$ ), $\delta$, ppm: 2.09 (s, 3H, $\mathrm{CH}_{3}$ ), 4.57 (s, 2H, $\left.\mathrm{NCH}_{2}\right), 5.56(\mathrm{~s}, 1 \mathrm{H}, \mathrm{CH}), 11.40$ (s, 1H, NH); ${ }^{13} \mathrm{C}$ NMR (DMSO- $\left.d_{6}\right), \delta$, ppm: 18.9, 43.5, 98.5, 151.7, 153.1, 162.8, 176.3. Anal. Calcd. for $\mathrm{C}_{7} \mathrm{H}_{7} \mathrm{~N}_{5} \mathrm{O}_{3}$ : C, 40.20; H, 3.37; N, 33.48. Found: C, 40.53; H, 3.57; N, 33.62.

\section{General procedure for the synthesis of carbamates (7-9) and (12-14)}

To a solution of azide $5(0.24 \mathrm{~g}, 1 \mathrm{mmol})$ in dry benzene $(3 \mathrm{~mL})$ or azide $6(0.21 \mathrm{~g}, 1 \mathrm{mmol})$ in dry dioxane $(3 \mathrm{~mL})$ the corresponding phenol $(1 \mathrm{mmol})$ was added. The reaction mixture was heated at reflux for $5 \mathrm{~h}$, then the solvent was removed under reduced pressure. The residue was 
worked up with diethyl ether to give a solid, which was collected by filtration and crystallized from the appropriate solvent.

Phenyl (6-methyl-2-methylthiopyrimidin-4-yloxy)methylcarbamate (7). Yield $0.18 \mathrm{~g}$ (59\%), mp 134-135 ${ }^{\circ} \mathrm{C}$ (2-propanol); IR, v, $\mathrm{cm}^{-1}$ : $1744(\mathrm{C}=\mathrm{O}), 3229(\mathrm{NH}) ;{ }^{1} \mathrm{H}$ NMR $\left(\mathrm{CDCl}_{3}\right), \delta$, ppm: $2.27\left(\mathrm{~s}, 3 \mathrm{H}, \mathrm{CH}_{3}\right), 2.59\left(\mathrm{~s}, 3 \mathrm{H}, \mathrm{SCH}_{3}\right), 5.50$ (d, J = 6.9 Hz, 2H, OCH$), 6.10(\mathrm{~s}, 1 \mathrm{H}, \mathrm{CH}) 6.53(\mathrm{t}$, $J=6.9 \mathrm{~Hz}, 1 \mathrm{H}, \mathrm{NH}), 7.15$ (d, $J=7.8 \mathrm{~Hz}, 2 \mathrm{H}, \mathrm{ArH}), 7.18-7.26$ (m, 1H, ArH), 7.34-7.40 (m, 2H, $\mathrm{ArH}) ;{ }^{13} \mathrm{C} \mathrm{NMR}\left(\mathrm{CDCl}_{3}\right), \delta$, ppm: 15.5, 24.1, 52.1, 108.0, 121.8, 125.9, 129.6, 150.9, 154.4, 161.5, 163.5, 163.9. Anal. Calcd. for $\mathrm{C}_{14} \mathrm{H}_{15} \mathrm{~N}_{3} \mathrm{O}_{3} \mathrm{~S}$ : C, 55.07; H, 4.95; N, 13.76. Found: C, 55.40; H, 5.09; N, 13.92 .

4-Chlorophenyl (6-methyl-2-methylthiopyrimidin-4-yloxy)methylcarbamate (8). Yield 0.16 $\mathrm{g}(48 \%)$, mp 141-142 ${ }^{\circ} \mathrm{C}$ (2-propanol); IR, v, cm ${ }^{-1}: 1737(\mathrm{C}=\mathrm{O}), 3191(\mathrm{NH}) ;{ }^{1} \mathrm{H}$ NMR $\left(\mathrm{CDCl}_{3}\right)$, $\delta$, ppm: $2.27\left(\mathrm{~s}, 3 \mathrm{H}, \mathrm{CH}_{3}\right), 2.59\left(\mathrm{~s}, 3 \mathrm{H}, \mathrm{SCH}_{3}\right), 5.50\left(\mathrm{~d}, J=6.6 \mathrm{~Hz}, 2 \mathrm{H}, \mathrm{OCH}_{2}\right), 6.10(\mathrm{~s}, 1 \mathrm{H}, \mathrm{CH})$ 6.56 (br s, $1 \mathrm{H}, \mathrm{NH}), 7.10$ (d, $J=8.4 \mathrm{~Hz}, 2 \mathrm{H}, \mathrm{ArH}), 7.33$ (d, $J=8.4 \mathrm{~Hz}, 2 \mathrm{H}, \mathrm{ArH}) ;{ }^{13} \mathrm{C}$ NMR $\left(\mathrm{CDCl}_{3}\right), \delta$, ppm: 15.5, 24.1, 52.1, 108.0, 123.1, 129.6, 131.2, 149.4, 154.0, 163.5, 164.0. Anal. Calcd. for $\mathrm{C}_{14} \mathrm{H}_{14} \mathrm{ClN}_{3} \mathrm{O}_{3} \mathrm{~S}$ : C, 49.49; H, 4.15; N, 12.37. Found: C, 49.21; H, 3.96; N, 12.21 .

4-Fluorophenyl (6-methyl-2-methylthiopyrimidin-4-yloxy)methylcarbamate (9). Yield 0.14 $\mathrm{g}(43 \%), \mathrm{mp} 148-149{ }^{\circ} \mathrm{C}$ (2-propanol); IR, v, cm ${ }^{-1}: 1737(\mathrm{C}=\mathrm{O}), 3234(\mathrm{NH}) ;{ }^{1} \mathrm{H} \mathrm{NMR}\left(\mathrm{CDCl}_{3}\right)$, $\delta$, ppm: $2.27\left(\mathrm{~s}, 3 \mathrm{H}, \mathrm{CH}_{3}\right), 2.59\left(\mathrm{~s}, 3 \mathrm{H}, \mathrm{SCH}_{3}\right), 5.51\left(\mathrm{~d}, J=6.9 \mathrm{~Hz}, 2 \mathrm{H}, \mathrm{OCH}_{2}\right), 6.10(\mathrm{~s}, 1 \mathrm{H}, \mathrm{CH})$ $6.53(\mathrm{t}, J=6.9 \mathrm{~Hz}, 1 \mathrm{H}, \mathrm{NH}), 7.0-7.17(\mathrm{~m}, 4 \mathrm{H}, \mathrm{ArH}) ;{ }^{13} \mathrm{C} \mathrm{NMR}\left(\mathrm{CDCl}_{3}\right), \delta, \mathrm{ppm}: 15.5,24.1$, 52.1, 108.0, 116.0, 116.3, 123.2, 123.3, 146.8, 154.3, 158.8, 161.4, 162.0, 163.5, 163.9. Anal. Calcd. for $\mathrm{C}_{14} \mathrm{H}_{14} \mathrm{FN}_{3} \mathrm{O}_{3} \mathrm{~S}$ : C, 52.00; H, 4.36; N, 13.00. Found: C, 52.33; H, 4.37; N, 13.21 .

Phenyl (6-methyl-2,4-dioxo-1,2-dihydropyrimidin-3(4H)-yl)methylcarbamate (12). Yield 0.2 g (74\%), mp 178-179 ${ }^{\circ} \mathrm{C}$ (ethanol); IR, v, $\mathrm{cm}^{-1}$ : 1646, $1721(\mathrm{C}=\mathrm{O}), 3268(\mathrm{NH}) ;{ }^{1} \mathrm{H}$ NMR (DMSO-d $)_{6}$ ), $\delta$, ppm: $2.06\left(\mathrm{~s}, 3 \mathrm{H}, \mathrm{CH}_{3}\right), 5.18\left(\mathrm{~d}, J=4.8 \mathrm{~Hz}, 2 \mathrm{H}, \mathrm{NCH}_{2}\right), 5.51(\mathrm{~s}, 1 \mathrm{H}, \mathrm{CH}) 7.10$ (d, $J=8.1 \mathrm{~Hz}, 2 \mathrm{H}, \mathrm{ArH}), 7.19-7.25$ (m, 4H, ArH), 7.36-7.42 (m, 2H, ArH), 8.17 (br s, 1H, NH), 11.18 (s, $1 \mathrm{H}, \mathrm{NH}) ;{ }^{13} \mathrm{C}$ NMR (DMSO-d $)$ ), $\delta$, ppm: 18.8, 46.8, 99.0, 122.5, 125.8, 130.0, 151.6, 151.8, 152.7, 154.4, 163.0. Anal. Calcd. for $\mathrm{C}_{13} \mathrm{H}_{13} \mathrm{~N}_{3} \mathrm{O}_{4}$ : C, 56.72; H, 4.76; N, 15.27. Found: C, 56.43; H, 4.93; N, 15.46.

4-Chlorophenyl (6-methyl-2,4-dioxo-1,2-dihydropyrimidin-3(4H)-yl)methylcarbamate (13). Yield $0.195 \mathrm{~g}(63 \%)$, mp 210-211 ${ }^{\circ} \mathrm{C}$ (ethanol); IR, $v, \mathrm{~cm}^{-1}: 1638,1725,1741(\mathrm{C}=\mathrm{O}), 3095$, $3321(\mathrm{NH}) ;{ }^{1} \mathrm{H}$ NMR (DMSO-d $), \delta$, ppm: 2.06 (s, 3H, $\left.\mathrm{CH}_{3}\right), 5.18\left(\mathrm{~d}, J=4.8 \mathrm{~Hz}, 2 \mathrm{H}, \mathrm{NCH}_{2}\right.$ ), $5.51(\mathrm{~s}, 1 \mathrm{H}, \mathrm{CH}) 7.15$ (d, J=8.4 Hz, 2H, ArH), 7.44 (d, J=8.4 Hz, 2H, ArH), 8.25 (br s, 1H, $\mathrm{NH}), 11.18$ (br s, $1 \mathrm{H}, \mathrm{NH}) ;{ }^{13} \mathrm{C}$ NMR (DMSO-d 6 ), $\delta$, ppm: 18.8, 46.8, 99.0, 124.4, 129.9, 141.6, 150.4, 151.8, 152.7, 154.1, 163.0. Anal. Calcd. for $\mathrm{C}_{13} \mathrm{H}_{12} \mathrm{ClN}_{3} \mathrm{O}_{4}$ : C, 50.42; H, 3.91; N, 13.57 . Found: C, 50.68; H, 4.27; N, 13.73 .

4-Fluorophenyl (6-methyl-2,4-dioxo-1,2-dihydropyrimidin-3(4H)-yl)methylcarbamate (14). Yield $0.205 \mathrm{~g}(70 \%)$, mp 197-198 ${ }^{\circ} \mathrm{C}$ (ethanol); IR, v, $\mathrm{cm}^{-1}: 1638,1736,1749(\mathrm{C}=\mathrm{O}), 3086$, 3271, $3442(\mathrm{NH}) ;{ }^{1} \mathrm{H}$ NMR (DMSO-d $)_{6}$, $\delta$, ppm: 2.06 (s, 3H, $\left.\mathrm{CH}_{3}\right), 5.18(\mathrm{~d}, J=4.8 \mathrm{~Hz}, 2 \mathrm{H}$, $\left.\mathrm{NCH}_{2}\right), 5.51(\mathrm{~s}, 1 \mathrm{H}, \mathrm{CH}) 7.11-7.24(\mathrm{~m}, 4 \mathrm{H}, \mathrm{ArH}), 8.21$ (br s, $\left.1 \mathrm{H}, \mathrm{NH}\right), 11.18(\mathrm{~s}, 1 \mathrm{H}, \mathrm{NH}) ;{ }^{13} \mathrm{C}$ NMR (DMSO-d ${ }_{6}$ ), $\delta$, ppm: 18.8, 46.8, 99.0, 116.3, 116.6, 124.2, 124.3, 147.7, 151.8, 152.7, 
154.4, 158.3, 161.5, 163.0. Anal. Calcd. for $\mathrm{C}_{13} \mathrm{H}_{12} \mathrm{FN}_{3} \mathrm{O}_{4}$ : C, 53.24; H, 4.12; N, 14.33. Found: C, 53.46; H, 4.45; N, 14.52 .

N-Benzyl-2-(6-methyl-2-methylthiopyrimidin-4-yloxy)acetamide (10). Benzylamine (0.107 g, $1 \mathrm{mmol})$ was added to a solution of azide $5(0.24 \mathrm{~g}, 1 \mathrm{mmol})$ in dry benzene $(5 \mathrm{~mL})$. The reaction mixture was heated at reflux for $1 \mathrm{~h}$, then cooled to room temperature. The resultant precipitate was collected by filtration, washed with methanol and crystallized from ethanol to yield 10, $0.192 \mathrm{~g}(63 \%), \mathrm{mp} 98-100{ }^{\circ} \mathrm{C}$; IR, v, cm ${ }^{-1}: 1654(\mathrm{C}=\mathrm{O}), 3287(\mathrm{NH}) ;{ }^{1} \mathrm{H} \mathrm{NMR}\left(\mathrm{CDCl}_{3}\right), \delta$, ppm: $2.40\left(\mathrm{~s}, 3 \mathrm{H}, \mathrm{CH}_{3}\right), 2.54\left(\mathrm{~s}, 3 \mathrm{H}, \mathrm{SCH}_{3}\right), 4.56\left(\mathrm{~d}, J=6 \mathrm{~Hz}, 2 \mathrm{H}, \mathrm{CH}_{2}\right), 4.95\left(\mathrm{~s}, 2 \mathrm{H}, \mathrm{OCH}_{2}\right), 6.35(\mathrm{~s}$, 1H, CH) 6.60 (br s, 1H, NH), 7.27-7.42 (m, 5H, ArH); ${ }^{13} \mathrm{C} \mathrm{NMR}\left(\mathrm{CDCl}_{3}\right), \delta$, ppm: 14.3, 24.1, 43.3, 65.0, 101.9, 128.0, 129.1, 137.9, 167.8, 167.9, 169.0, 172.2. Anal. Calcd. for $\mathrm{C}_{15} \mathrm{H}_{17} \mathrm{~N}_{3} \mathrm{O}_{2} \mathrm{~S}$ : C, 59.38; H, 5.65; N, 13.85. Found: C, 59.63; H, 5.66; N, 13.97.

Methyl (6-methyl-2,4-dioxo-1,2-dihydropyrimidin-3(4H)-yl)methylcarbamate (11). The solution of azide $6(0.21 \mathrm{~g}, 1 \mathrm{mmol})$ in abs. methanol $(2 \mathrm{~mL})$ was heated at reflux for $5 \mathrm{~h}$ and the reaction mixture was evaporated under reduced pressure to dryness. The residue was crystallized from 2-propanol to yield 11, $0.12 \mathrm{~g}(56 \%), \mathrm{mp} \mathrm{189-191}{ }^{\circ} \mathrm{C}$; IR, v, $\mathrm{cm}^{-1}$ : 1642, $1734(\mathrm{C}=\mathrm{O})$, 3092, $3426(\mathrm{NH}) ;{ }^{1} \mathrm{H}$ NMR (DMSO-d $)$, $\delta$, ppm: 2.04 (s, 3H, $\left.\mathrm{CH}_{3}\right), 3.53$ (s, 3H, OCH $), 5.09$ (d, $\left.J=6 \mathrm{~Hz}, 2 \mathrm{H}, \mathrm{NCH}_{2}\right), 5.46(\mathrm{~s}, 1 \mathrm{H}, \mathrm{CH}), 7.49($ br s, $1 \mathrm{H}, \mathrm{NH}), 11.13(\mathrm{~s}, 1 \mathrm{H}, \mathrm{NH}) ;{ }^{13} \mathrm{C} \mathrm{NMR}$ (DMSO- $d_{6}$ ), $\delta$, ppm: 18.8, 46.8, 52.2, 99.0, 151.8, 152.6, 156.5, 163.0. Anal. Calcd. for $\mathrm{C}_{8} \mathrm{H}_{11} \mathrm{~N}_{3} \mathrm{O}_{4}$ : C, 45.07; H, 5.20; N, 19.71. Found: C, 45.34; H, 5.49; N, 19.59 .

\section{General procedure for the synthesis of ureas (15-20)}

To a solution of azide $6(0.21 \mathrm{~g}, 1 \mathrm{mmol})$ in dry dioxane $(7 \mathrm{~mL})$ the corresponding aniline (1 mmol) was added. The reaction mixture was heated at reflux for $1 \mathrm{~h}$ and cooled to room temperature. The resultant precipitate was collected by filtration, washed with methanol and crystallized from ethanol.

$\boldsymbol{N}$-[(6-Methyl-2,4-dioxo-1,2-dihydropyrimidin-3(4H)-yl)methyl]- $\boldsymbol{N}^{\prime}$-phenylurea (15). Yield $0.24 \mathrm{~g}(88 \%), \operatorname{mp} 227-229{ }^{\circ} \mathrm{C}$; IR, $v, \mathrm{~cm}^{-1}: 1644,1682,1730(\mathrm{C}=\mathrm{O}), 3204,3263,3318,3419$ $(\mathrm{NH}) ;{ }^{1} \mathrm{H}$ NMR (DMSO-d $), \delta$, ppm: $2.05\left(\mathrm{~s}, 3 \mathrm{H}, \mathrm{CH}_{3}\right), 5.24\left(\mathrm{~d}, J=6 \mathrm{~Hz}, 2 \mathrm{H}, \mathrm{NCH}_{2}\right), 5.51$ (s, $1 \mathrm{H}, \mathrm{CH})$ 6.78-7.04 (m, 2H, NH, ArH), 7.16-7.32 (m, 2H, ArH), 7.32-7.48 (m, 2H, ArH), 8.75 (s, $1 \mathrm{H}, \mathrm{NH}), 11.21(\mathrm{~s}, 1 \mathrm{H}, \mathrm{NH}) ;{ }^{13} \mathrm{C} \mathrm{NMR}\left(\mathrm{DMSO}-\mathrm{d}_{6}\right), \delta$, ppm: 18.8, 45.8, 99.1, 118.3, 122.1, 129.4, 140.8, 151.9, 152.9, 154.7, 163.4. Anal. Calcd. for $\mathrm{C}_{13} \mathrm{H}_{14} \mathrm{~N}_{4} \mathrm{O}_{3}: \mathrm{C}, 56.93 ; \mathrm{H}, 5.14 ; \mathrm{N}$, 20.43. Found: C, 56.61; H, 5.38; N, 20.29.

$\boldsymbol{N}$-[(6-Methyl-2,4-dioxo-1,2-dihydropyrimidin-3(4H)-yl)methyl]- $N^{\prime}$-(2-methylphenyl)urea (16). Yield $0.173 \mathrm{~g}(60 \%), \mathrm{mp} 194-195{ }^{\circ} \mathrm{C}$; IR, v, $\mathrm{cm}^{-1}$ : 1648, 1677, 1729 (C=O), 3181, 3318, $3428(\mathrm{NH}) ;{ }^{1} \mathrm{H}$ NMR (DMSO-d $), \delta$, ppm: 2.06 (s, 3H, $\left.\mathrm{CH}_{3}\right), 2.17$ (s, 3H, $\left.\mathrm{CH}_{3}\right), 5.25$ (d, $J=5.4$ $\left.\mathrm{Hz}, 2 \mathrm{H}, \mathrm{NCH}_{2}\right), 5.52(\mathrm{~s}, 1 \mathrm{H}, \mathrm{CH})$ 6.82-6.95 (m, 1H, ArH), 7.02-7.21 (m, 2H, NH, ArH), 7.307.47 (m, 1H, ArH), $7.84(\mathrm{~d}, J=7.8 \mathrm{~Hz}, 1 \mathrm{H}, \mathrm{ArH}), 7.94(\mathrm{~s}, 1 \mathrm{H}, \mathrm{NH}), 11.20(\mathrm{~s}, 1 \mathrm{H}, \mathrm{NH}) ;{ }^{13} \mathrm{C}$ NMR (DMSO-d (D) $\delta$, ppm: 18.6, 18.8, 45.7, 99.0, 121.0, 122.7, 126.7, 127.3 130.7, 138.5, 151.9, 152.8, 154.7, 163.3. Anal. Calcd. for $\mathrm{C}_{14} \mathrm{H}_{16} \mathrm{~N}_{4} \mathrm{O}_{3}$ : C, 58.32; H, 5.59; N, 19.43. Found: C, 58.03; H, 5.59; N, 19.27. 
$\boldsymbol{N}$-(4-Methoxyphenyl)- $\boldsymbol{N}$ '-[(6-methyl-2,4-dioxo-1,2-dihydropyrimidin-3(4H)-yl)methyl]urea (17). Yield $0.21 \mathrm{~g}(69 \%), \mathrm{mp} 230-231{ }^{\circ} \mathrm{C}$; IR, $v, \mathrm{~cm}^{-1}$ : 1645, 1711, $1740(\mathrm{C}=\mathrm{O}), 3104,3171$, $3312(\mathrm{NH}) ;{ }^{1} \mathrm{H}$ NMR (DMSO-d $\left.)_{6}\right), \delta$, ppm: $2.05\left(\mathrm{~s}, 3 \mathrm{H}, \mathrm{CH}_{3}\right), 2.52\left(\mathrm{~s}, 3 \mathrm{H}, \mathrm{OCH}_{3}\right), 5.22(\mathrm{~d}, J=$ $\left.6.6 \mathrm{~Hz}, 2 \mathrm{H}, \mathrm{NCH}_{2}\right), 5.51(\mathrm{~s}, 1 \mathrm{H}, \mathrm{CH}) 6.78(\mathrm{t}, J=6.6 \mathrm{~Hz}, 1 \mathrm{H}, \mathrm{NH}), 6.83(\mathrm{~d}, J=8.7 \mathrm{~Hz}, 2 \mathrm{H}, \mathrm{ArH})$, $7.27(\mathrm{~d}, J=8.7 \mathrm{~Hz}, 2 \mathrm{H}, \mathrm{ArH}), 8.56(\mathrm{~s}, 1 \mathrm{H}, \mathrm{NH}), 11.19(\mathrm{~s}, 1 \mathrm{H}, \mathrm{NH}) ;{ }^{13} \mathrm{C}$ NMR (DMSO-d 6 ), $\delta$, ppm: 18.8, 45.8, 55.8, 99.0, 114.6, 120.0, 133.9, 151.9, 152.2, 152.8, 154.8, 163.4. Anal. Calcd. for $\mathrm{C}_{14} \mathrm{H}_{16} \mathrm{~N}_{4} \mathrm{O}_{4}$ : C, 55.26; H, 5.30; N, 18.41. Found: C, 55.47; H, 5.53; N, 18.58 .

$\boldsymbol{N}$-(4-Fluorophenyl)- $\boldsymbol{N}^{\prime}$-[(6-methyl-2,4-dioxo-1,2-dihydropyrimidin-3(4H)-yl)methyl]urea (18). Yield 0.254g (87\%), mp 231-233 ${ }^{\circ} \mathrm{C}$; IR, v, $\mathrm{cm}^{-1}: 1638,1682,1732(\mathrm{C}=\mathrm{O}), 3202,3279$, $3416(\mathrm{NH}) ;{ }^{1} \mathrm{H}$ NMR (DMSO-d $)$, $\delta$, ppm: 2.05 (s, 3H, $\left.\mathrm{CH}_{3}\right), 5.23\left(\mathrm{~d}, J=6 \mathrm{~Hz}, 2 \mathrm{H}, \mathrm{NCH}_{2}\right), 5.51$ $(\mathrm{s}, 1 \mathrm{H}, \mathrm{CH}) 6.87$ (t, $J=6 \mathrm{~Hz}, 1 \mathrm{H}, \mathrm{NH}), 7.02-7.16(\mathrm{~m}, 2 \mathrm{H}, \mathrm{ArH}), 7.30-7.47$ (m, 2H, ArH), 8.79 $(\mathrm{s}, 1 \mathrm{H}, \mathrm{NH}), 11.21(\mathrm{~s}, 1 \mathrm{H}, \mathrm{NH}) ;{ }^{13} \mathrm{C} \mathrm{NMR}$ (DMSO-d $), \delta$, ppm: 18.8, 45.8, 99.1, 115.7, 116.1, $119.9,120.0,137.1,137.2,151.9,152.9,154.7,156.2,159.3,163.4$. Anal. Calcd. for $\mathrm{C}_{13} \mathrm{H}_{13} \mathrm{FN}_{4} \mathrm{O}_{3}$ : C, 53.42; H, 4.48; N, 19.17. Found: C, 53.71; H, 4.79; N, 19.31.

$\boldsymbol{N}$-(2,4-Dichlorophenyl)- $\boldsymbol{N}^{\prime}$-[(6-methyl-2,4-dioxo-1,2-dihydropyrimidin-3(4H)-

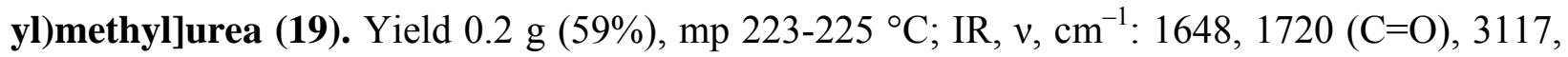
3177, $3339(\mathrm{NH})$; ${ }^{1} \mathrm{H}$ NMR (DMSO-d $)$, $\delta$, ppm: 2.05 (s, 3H, $\left.\mathrm{CH}_{3}\right), 5.24$ (d, $J=6 \mathrm{~Hz}, 2 \mathrm{H}$, $\left.\mathrm{NCH}_{2}\right), 5.51(\mathrm{~s}, 1 \mathrm{H}, \mathrm{CH}) 7.33(\mathrm{~d}, J=9 \mathrm{~Hz}, 1 \mathrm{H}, \mathrm{ArH}), 7.54(\mathrm{~s}, 1 \mathrm{H}, \mathrm{ArH}), 7.86(\mathrm{t}, J=6 \mathrm{~Hz}, 1 \mathrm{H}$, $\mathrm{NH}), 8.18(\mathrm{~d}, J=9 \mathrm{~Hz}, 1 \mathrm{H}, \mathrm{ArH}), 8.45(\mathrm{~s}, 1 \mathrm{H}, \mathrm{NH}), 11.21(\mathrm{~s}, 1 \mathrm{H}, \mathrm{NH}) ;{ }^{13} \mathrm{C}$ NMR (DMSO- $\left.d_{6}\right), \delta$, ppm: 18.8, 45.5, 99.0, 122.6, 122.8, 126.2, 128.1 129.1, 136.4, 151.9, 152.2, 154.4, 163.3. Anal. Calcd. for $\mathrm{C}_{13} \mathrm{H}_{12} \mathrm{Cl}_{2} \mathrm{~N}_{4} \mathrm{O}_{3}$ : C, 45.50; H, 3.52; N, 16.33. Found: C, 45.55; H, 3.77; N, 16.45 .

$N$-[(6-Methyl-2,4-dioxo-1,2-dihydropyrimidin-3(4H)-yl)methyl]- $N^{\prime}-[3-$

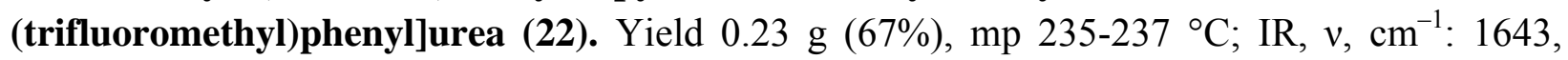
1689, $1723(\mathrm{C}=\mathrm{O}), 3121,3166,3393(\mathrm{NH}) ;{ }^{1} \mathrm{H}$ NMR (DMSO-d $), \delta$, ppm: $2.05\left(\mathrm{~s}, 3 \mathrm{H}, \mathrm{CH}_{3}\right)$, $5.25\left(\mathrm{~d}, J=6.3 \mathrm{~Hz}, 2 \mathrm{H}, \mathrm{NCH}_{2}\right), 5.52(\mathrm{~s}, 1 \mathrm{H}, \mathrm{CH}), 7.01(\mathrm{t}, J=6.3 \mathrm{~Hz}, 1 \mathrm{H}, \mathrm{NH}), 7.26(\mathrm{~d}, J=6.9$ $\mathrm{Hz}, 1 \mathrm{H}, \mathrm{ArH}), 7.38-7.56$ (m, 2H, ArH), 7.98 (s, 1H, ArH), 9.13 (s, 1H, NH), 11.23 (s, 1H, NH); ${ }^{13} \mathrm{C}$ NMR (DMSO-d $)$ ), $\delta$, ppm: 18.8, 45.6, 99.1, 114.2, 118.4, 121.9, 123.1 126.7, 130.0, 130.4, 130.6, 141.6, 151.9, 152.9, 154.6, 163.4. Anal. Calcd. for $\mathrm{C}_{14} \mathrm{H}_{13} \mathrm{~F}_{3} \mathrm{~N}_{4} \mathrm{O}_{3}: \mathrm{C}, 49.13 ; \mathrm{H}, 3.83 ; \mathrm{N}$, 16.37. Found: C, 49.39; H, 3.90; N, 16.58 .

$\boldsymbol{N}, \boldsymbol{N}^{\prime}$-Bis-[(6-methyl-2,4-dioxo-1,2-dihydropyrimidin-3(4H)-yl)methyl]urea (21). Water $(0.018 \mathrm{~g}, 1 \mathrm{mmol})$ was added to a solution of azide $6(0.21 \mathrm{~g}, 1 \mathrm{mmol})$ in dioxane $(7 \mathrm{~mL})$. The reaction mixture was heated at reflux for $4 \mathrm{~h}$ and cooled to room temperature. The resultant precipitate was collected by filtration, washed with methanol and crystallized from a mixture of dimethyl sulfoxide-water to yield 21, $0.2 \mathrm{~g}(59 \%), \mathrm{mp} \mathrm{312-314}{ }^{\circ} \mathrm{C}$; IR, $v, \mathrm{~cm}^{-1}$ : 1647,1715 $(\mathrm{C}=\mathrm{O}), 3112,3187,3250,3383(\mathrm{NH}) ;{ }^{1} \mathrm{H}$ NMR (DMSO-d $), \delta$, ppm: 2.03 (s, 6H, 2CH $\mathrm{CH}_{3}, 5.10$ (d, $\left.J=6.3 \mathrm{~Hz}, 4 \mathrm{H}, 2 \mathrm{NCH}_{2}\right), 5.46(\mathrm{~s}, 2 \mathrm{H}, 2 \mathrm{CH}) 6.96(\mathrm{t}, J=6.3 \mathrm{~Hz}, 2 \mathrm{H}, 2 \mathrm{NH}), 11.12(\mathrm{~s}, 2 \mathrm{H}, 2 \mathrm{NH})$;

${ }^{13} \mathrm{C}$ NMR (DMSO- $\left.d_{6}\right), \delta$, ppm: 18.8, 45.9, 99.1, 151.8, 152.7, 156.2, 163.3. Anal. Calcd. for $\mathrm{C}_{13} \mathrm{H}_{16} \mathrm{~N}_{6} \mathrm{O}_{5}$ : C, 46.43; H, 4.80; N, 24.99. Found: C, 46.30; H, 5.09; N, 25.07. 


\section{Acknowledgements}

The authors thank Dr. J. M. Padrón and co-workers from BIOLAB, Instituto Canario de Investigación del Cancer, C/ Astrofisico Francisco Sanchez, La Laguna, Spain for the in vitro evaluation of the antiproliferative activity.

\section{References}

1. Pozharskii, A. F.; Soldatenkov, A. T.; Katrizky, A. R. Heterocycles in Life and Society. Wiley: New York, 1997.

2. Delaney, J.; Clarke, E.; Hughes, D.; Rice, M. Drug Discov. Today 2006, 11, 839.

3. Jain, K. S.; Chitre, T. S.; Miniyar, P. B.; Kathiravan, M. K.; Bendre, V. S.; Veer, V. S.; Shahane, S. R.; Shishoo, C. J. Curr. Sci. 2006, 90, 793.

4. Miwa, M.; Ura, M.; Nishida, M.; Sawada, N.; Ishikawa, T.; Mori, K.; Shimma, N.; Umeda, I.; Ishitsuka, H. Eur. J. Cancer 1998, 34, 1274.

5. Falcao, E. P. S.; Melo, S. J.; Srivastava, R. M.; Catanho, M. T. J.; Nascimento, S. C. Eur. J. Med. Chem. 2006, 41, 276.

6. Jakubkiene, V.; Burbuliene, M. M.; Udrenaite, E.; Garaliene, V.; Vainilavicius, P. Pharmazie 2002, 57, 610.

7. Shao, L.; Zhou, X.; Jin, Z.; Fang, J. X. Heteroatom Chem. 2008, 19, 2.

8. Benchabane, Y.; Di Giorgio, C.; Boyer, G.; Sabatier, A. S.; Allegro, D.; Peyrot, V.; De Meo, M. Eur. J. Med. Chem. 2009, 44, 2459.

9. Pirisi, M. A.; Murineddu, J. M.; Mussinu, J. M.; Pinna, G. A. Il Farmaco 2002, 57, 331.

10. Borrel, Ch.; Thoret, S.; Cachet, X.; Guenard, D.; Tilleguin, F.; Koch, M.; Michel, S. Bioorg. Med. Chem. 2005, 13, 3853.

11. Sanmartin, C.; Echeveria, M.; Mendivil, B.; Corden, L.; Cubedo, E.; Garcia-Foncillas, J.; Font, M.; Palop, J. A. Bioorg. Med. Chem, 2005, 13, 2031.

12. Kakadiya, R.; Dong, H.; Lee, P. C.; Kapuriya, N.; Zhang, X.; Chou, T. C.; Lee, T. C.; Kapuriya, K.; Shah, A.; Su, T. L. Bioorg. Med. Chem. 2009, 17, 5614.

13. Latthe, P. R.; Shinge, P. S.; Badami, B. V.; Patil, P. B.; Holihosur, S. N. J. Chem. Sci. 2006, 118, 249.

14. Mor, M.; Lodola, A.; Rivara, S.; Vacondio, F.; Duranti, A.; Tontini, A.; Sanchini, S.; Piersanti, G.; Clapper, J. R.; King, A. R.; Tarzia, G.; Piomelli, D. J. Med. Chem. 2008, 51, 3487.

15. Burns, C. J.; Harte, M. F.; Bu, X.; Fantino, E.; Joffe, M.; Sikanyika, H.; Su, S.; Tranberg, C. E.; Wilson, N.; Charman, S. A.; Shackleford, D. M.; Wilks, A. F. Bioorg. Med. Chem. Lett. 2009, 19, 4639.

16. Tao, Z. F.; Chen, Z.; Bui, M. H.; Kovar, P.; Johnson, E.; Bouska, J.; Zhang, H.; Rosenberg, S.; Sowin, T.; Lin, N. H. Bioorg. Med. Chem. Lett. 2007, 23, 6593. 
17. Brugel, T. A.; Maier, J. A.; Clark, M. P.; Sabat, M.; Golebiowski, A.; Bookland, R. G.; Laufersweiler, M. J.; Laughlin, S. K.; VanRens, J. C.; De, B.; Hsieh, L. C.; Mekel, M. J.; Janusz, M. J. Bioorg. Med. Chem. Lett. 2006, 16, 3510.

18. Maier, J. A.; Brugel, T. A.; Clark, M. P.; Sabat, M.; Golebiowski, A.; Bookland, R. G.; Laufersweiler, M. J.; Laughlin, S. K.; VanRens, J. C.; De, B.; Hsieh, L. C.; Brown, K. K.; Juergens, K.; Walter, R. L.; Janusz, M. J. Bioorg. Med. Chem. Lett. 2006, 16, 3514.

19. Maier, J. A.; Brugel, T. A.; Sabat, M.; Golebiowski, A.; Laufersweiler, M. J.; VanRens, J. C.; Hopkins, C. R.; De, B.; Hsieh, L. C.; Brown, K. K.; Easwaran, V.; Janusz, M. J. Bioorg. Med. Chem. Lett. 2006, 16, 3646.

20. Kim, H. S.; Kim, T, E.; Lee, S. U.; Kim, D. I.; Han, S. W.; Okamoto, Y.; Mitomi, T.; Kurasawa, Y. J. Heterocyclic Chem. 1998, 35, 1515.

21. Zanirato, P. Arkivoc 2009, (i), 97.

22. Katritzky, A. R.; Widyan, K.; Kirichenko, K. J. Org. Chem. 2007, 72, 5802.

23. Papeo, G.; Posteri, H.; Vianello, P.; Varassi, M. Synthesis 2004, 17, 2886.

24. Rumi, L.; Pfleger, C.; Spurr, P.; Klinkhammer, U.; Bannwarth, W. Org. Process Res. Dev. 2009, 13, 747.

25. Jakubkiene, V.; Burbuliene, M. M.; Mekuskiene, G.; Udrenaite, E.; Gaidelis, P.; Vainilavicius, P. Il Farmaco 2003, 58, 323.

26. Jakubkiene, V.; Stura, A.; Vainilavicius, P. Khim. Geterotsikl. Soed. 2004, 918.

27. Vainilavicius, P.; Sedereviciute, V.; Mociskyte, S.; Khim. Geterotsikl. Soed. 1992, 1655.

28. Vainilavicius, P.; Smicius, R.; Jakubkiene, V.; Tumkevicius, S. Monatsh. Chem. 2001, 132, 825. 